\title{
Asymptomatic giant congenital left atrial aneurysm
}

\author{
Kahraman Yakut, Birgül Varan, İlkay Erdoğan \\ Department of Pediatric Cardiology, Baskent University Faculty of Medicine, Ankara, Turkey. \\ E-mail:kahramanyakut@gmail.com \\ Received: 22nd January 2017, Accepted: 4th November 2017
}

SUMMARY: Yakut K, Varan B, Erdoğan I. Asymptomatic giant congenital left
atrial aneurysm. Turk J Pediatr 2019; 61: 117-119.
Congenital aneurysm of the left atrial appendage can be caused by congenital
dysplasia of the pectinate muscles and may be accompanied by a congenital
absence of the pericardium. Symptoms generally manifest after two decades
and the most common symptom is atrial arrhythmia in the form of atrial
fibrillation / flutter. A four year-old patient with no symptoms underwent
an echocardiographic examination for the investigation of a heart murmur.
Echocardiographic examination revealed a large cystic lesion occupying the left
hemithorax and compressing the left ventricle. The patient was referred to
our center. The lesion caused displacement of the heart rightward behind the
sternum which made the examination difficult. We aimed to present this rare
case of giant left atrial appendage aneurysm in the light of current literature.

Key words: congenital, atrial appendage, complication, aneurysm.

Congenital left atrial appendage aneurysm is a very rare form of congenital heart disease with potentially lethal complications. ${ }^{1-4}$ The most common complications are thromboembolic events and supraventricular tachyarrhythmias. ${ }^{1,4,5}$ This condition is usually asymptomatic until the second or third decade of life. $1,2,4,6$ We report a rare case of asymptomatic congenital giant aneurysm of the left atrial appendage, leading to left ventricular compression and moderate-severe mitral valve regurgitation.

\section{Case Report}

A four-year-old boy underwent cardiologic examination after a murmur was detected during a routine physical examination. Echocardiographic examination performed at another hospital was reported to be suboptimal due to a large cystic lesion in the paracardiac area. To evaluate the pericardial cystic lesion reported on echocardiography, thoracic CT examination was performed. Thoracic computed tomography revealed a large cystic lesion in the paracardiac area, and the patient was referred to our clinic for further investigation. On physical examination, there was no pathological finding other than a pansystolic grade II/VI murmur on the $4^{\text {th }}-5^{\text {th }}$ intercostal space to the left of sternum. A chest radiogram showed a double density sign at the right heart margin, carina angle $>90$ degrees, convex left atrial appendage, and enlargement at the left heart margin (Fig. 1). An electrocardiogram showed a heart rate of 112 beats/min in normal sinus rhythm. There were no reports of arrhythmia or thromboembolism episode. Echocardiographic examination revealed a giant aneurysm in the left atrial appendage occupying the left hemithorax and compressing the left ventricle, mitral valve insufficiency, causing a shift of the heart to the right behind the sternum, which interfered with optimal imaging, (Fig. 2. left atrium $56 \times 116$ millimeters). The patient had severe mitral insufficiency. There was no thrombus in the congenital left atrial appendage aneurysm, and the patient was put on antiplatelet medication with acetylsalicylic acid and was scheduled for surgery. To determine the extent of left atrial appendage aneurysm and associated additional lesions prior to surgery magnetic resonance (MR) imaging was performed. Left atrial appendage aneurysm before surgery was imaged with MR angiography (Fig. 3-4. left atrium 56.7 x84.1 millimeters). Written informed consent was obtained from the parents of the patient. 


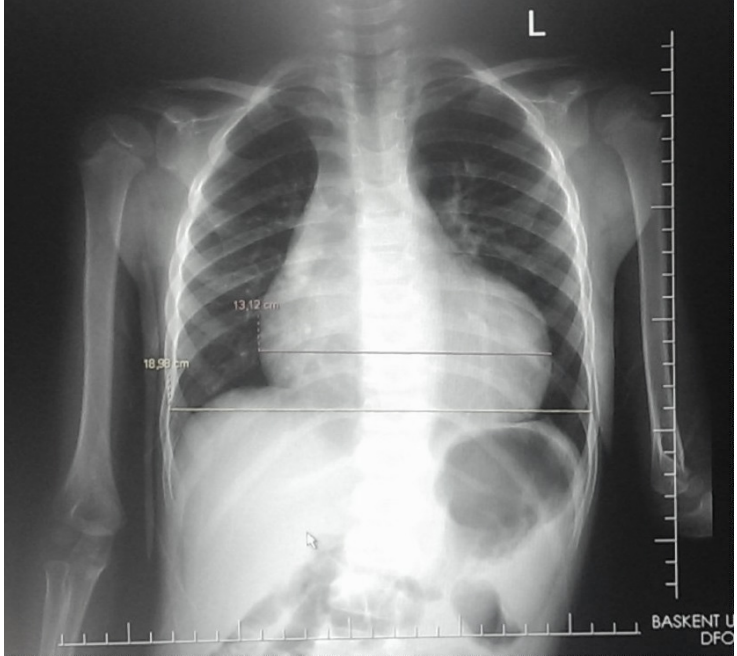

Fig. 1. Chest radiogram of giant left atrial appendage.

\section{Discussion}

Congenital aneurysms of the left atrium and left atrial appendage are extremely rare, and there are only limited case reports in literature about the definition and management of the condition. ${ }^{4,7}$ Morphologically, the genesis of the congenital left atrial appendage aneurysm has been attributed to congenital dysplasia of pectinate muscles, ${ }^{1}$ while a congenital absence of pericardium has also been correlated with this anomaly. 4,7 The condition is frequently accompanied by mitral valve insufficiency. Left atrial dilation caused by the aneurysm can result in mitral annular deformity, which in turn may lead to mitral valve insufficiency. Although the lesions are small in the beginning, they are thought to grow and reach a significant size

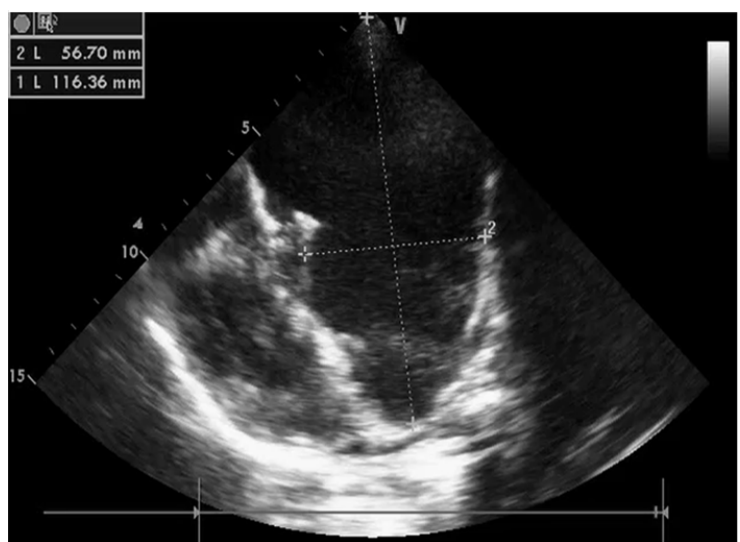

Fig. 2. Two-dimensional echocardiographic four-chamber view, showing the giant aneurysm originating from the left atrium.
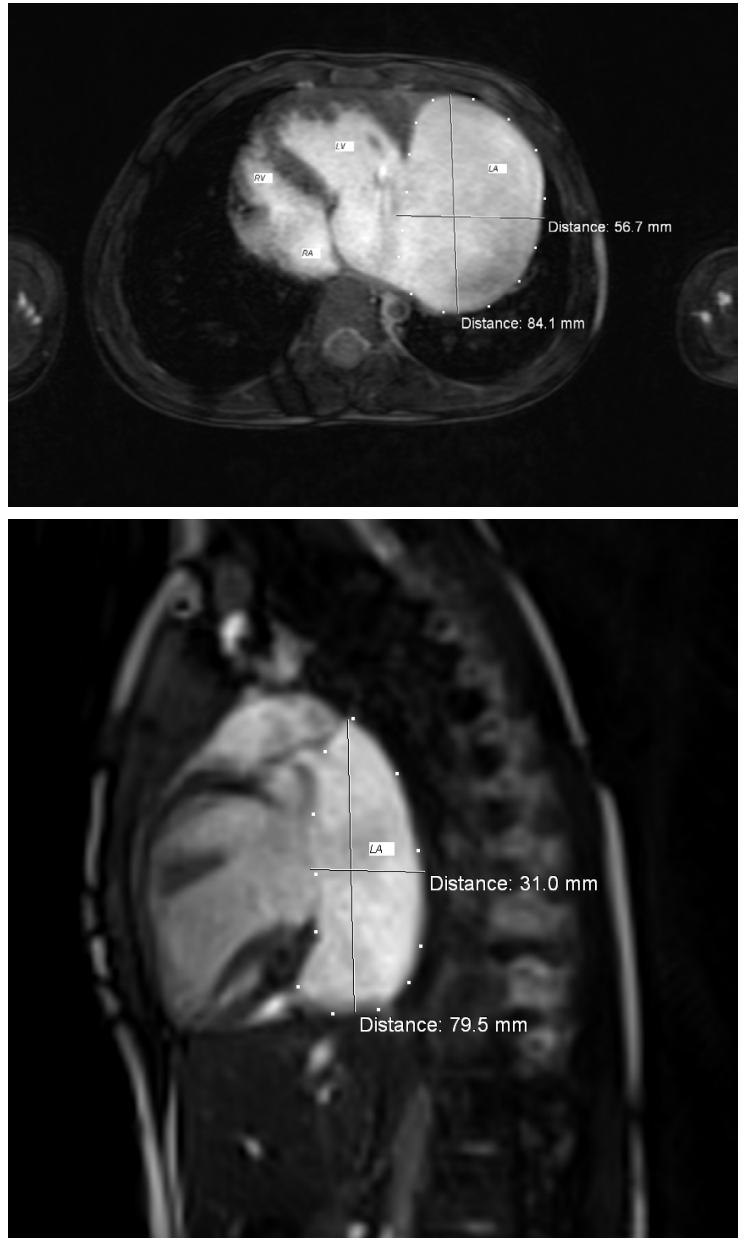

Fig. 3, 4. Left atrial appendage aneurysm image with magnetic resonance angiography: left ventricle (LV), right ventricle $(\mathrm{RV})$, right atrium (RA), giant left atrial appendage aneurysm (LA).

over years. Congenital left atrial appendage aneurysm is often asymptomatic in childhood, and the diagnosis may be delayed until adulthood. The symptoms often begin after the second decade, and the most common presenting symptoms are atrial arrhythmias as atrial fibrillation and flutter, 8 although thromboembolic events and supraventricular arrhythmias are also commonly reported. ${ }^{2-7}$ Thrombus formation has been reported in the aneurysm in one-third of patients with congenital left atrial appendage aneurysm, ${ }^{8}$ which may cause episodes of chest pain as a result of the compression of the left coronary artery and its branches. ${ }^{9}$ The risk of complications is reported to increase in parallel to the size of the aneurysm. ${ }^{8,10}$ Invasive and non-invasive diagnostic methods can be used 
in congenital left atrial appendage aneurysm, such as transthoracic echocardiography, transesophageal echocardiography, thoracic computed tomography, magnetic resonance imaging and angiography. ${ }^{8}$ Transthoracic echocardiography is the most useful noninvasive method for the examination of congenital left atrial appendage aneurysm, while transesophageal echocardiography is also useful, but more invasive in detecting the presence of a thrombus in the left atrium or a left atrial aneurysm. This technique must be reserved for the detection of accompanying complex cardiac pathologies in which the angiography, echocardiography or magnetic resonance imaging may fail. Although catheter angiography has been considered the gold standard in evaluating the anatomy of coronary arteries when a compression to the left coronary artery and its branches is suspected, a noninvasive coronary computed tomography angiography can also provide results with high accuracy within a short time. ${ }^{11,12}$ There are few reports of pediatric cases diagnosed with echocardiography. Congenital left atrial appendage aneurysm can mimic tumors or cystic structures as a result of abnormal cardiac margins observed in routine chest radiograms or thoracic computed tomography images. The present case was also reported to have a large cystic lesion in the paracardiac area, based on a thoracic computed tomography and echocardiographic examination. Mediastinal mass lesions, pericardial cysts, bronchogenic cysts, epicardial lipoma, cardiac and paracardiac tumors, pericardial defects and valvular diseases must be considered in differential diagnosis. The basic treatment method involves surgical resection of the left atrial appendage aneurysm via median sternotomy under cardiopulmonary bypass. Surgical management has been reported to reduce dyspnea, dysrhythmia, heart failure, thromboembolic events and the risk of rupture. ${ }^{10}$

This rare condition must be considered in differential diagnosis of cardiac lesions neighboring the left heart borders. Early diagnosis and surgical treatment is recommended in order to reduce complications, mortality and morbidity.

\section{REFERENCES}

1. Chowdhury UK, Seth S, Govindappa R, Jagia P, Malhotra P. Congenital left atrial appendage aneurysm: A case report and brief review of literature. Heart Lung Circ 2009; 18: 412-416.

2. Mathur A, Zehr KJ, Sinak LJ, Rea RF. Left atrial appendage aneurysm. Ann Thorac Surg 2005; 79: 1392-1393.

3. Acarturk E, Kanadasi M, Yerdelen VD, Akpinar O, Ozeren A, Saygili OB. Left atrial appendage aneurysm presenting with recurrent embolic strokes. Int J Cardiovasc Imaging 2003; 19: 495-497.

4. Conradi G, Deetjen A, Mollmann S, Hamm CW, Dill T. Symptomatic atrial fibrillation as the first symptom of a giant left atrial appendage aneurysm. Clin Res Cardiol 2006; 95: 614-616.

5. Zhang PF, Zhang M, Zhang W, Yao GH, Wu SM, Zhang Y. Giant aneurysm of the left atrial appendage: Detected by real-time 3-dimensional echocardiography. Tex Heart Inst J 2010; 37: 129-130.

6. Lekkerkerker JC, Jaarsma W, Cramer MJ. Congenital giant aneurysm of the left atrial appendage. Heart 2005; 91: e21.

7. Kunishima T, Musha H, Yamamoto T, et al. Congenital giant aneurysm of the left atrial appendage mimicking pericardial absence. Jpn Circ J 2001; 65: 56-59.

8. Park JS, Lee DH, Han SS, et al. Incidentally found, growing congenital aneurysm of the left atrium. J Korean Med Sci 2003; 18: 262-266.

9. Gupta S, Agarwal S, Pratap H, Datt V, Banerjee A. Congenital aneurysm of left atrial appendage: A case report. J Card Surg 2010: 25: 37-40.

10. Cho MJ, Park JA, Lee HD, Choo KS, Sung SC. Congenital left atrial appendage aneurysm diagnosed by fetal echocardiography. J Clin Ultrasound 2010; 38 : 94-96.

11. Plonska-Gosciniak E, Larysz B, Jurczyk K, Kasprzak JD. Five-chambered heart: A 20-year story of left atrial appendage aneurysm. Eur Heart J 2009; 30: 1014.

12. Smeglin A, Merchan J, Maysky M, Johnstone M, Pastore JO. Images in cardiovascular medicine: Giant left atrial appendage aneurysm. Circulation 2008; 118: 2393-2394. 